Wawilow's measurements using linearly polarised light for excitation and observing along the normal to the plane containing the direction of propagation and the direction of vibration of the exciting light. Though our values differ considerably from those calculated from Wawilow's results (the polarisation varies also with the concentration of the dye-stuff), the general nature of the variation of polarisation with the exciting wave-length, and also its negative value for excitation by $\lambda 3125-31$, are fully confirmed. The change in sign of the polarisation in the case of fluorescein solution occurring when the exciting wave-length is changed from $\lambda 4358$ to $\lambda 3125-31$, is seen clearly in Fig. $I(a)$ and $(b)$, which are photographs of the fluorescent radiations excited by $\lambda 4358$ and $\lambda 3125-31$ respectively, taken through a suitably oriented Savart plate and analyser. The images of a fixed cross-wire which appear in the photographs, serve as reference for the positions of the fringes. It is easily seen that whereas in Fig. $1(a)$ the centre of the cross-wire falls on a bright fringe, it falis on a dark fringe in Fig. $1(b)$.

Also observations along different directions show for $\lambda .3125-31$ excitation that the intensity of the fluorescent radiations vibrating normal to the direction of the vibrations of the exciting light is always greater than that of fluorescent vibrations along the direction of the exciting vibrations.

K. S. Krishnan.

S. M. Mitra.

Physics Laboratory,

University of Dacca.

Dec. 23.

${ }^{1}$ Z. Phys., 55, $6 \mathrm{c} 0 ; 1929$.

\section{Synthesis of Chrysene and Certain Derivatives}

THE very interesting letter of Messrs. Cook, Dodds and Hewett ${ }^{1}$ on the subject of the cestrus-producing action of a keto-tetrahydrophenanthrene contains a statement that experiments are being made with the object of converting this ketone into ketohydroxycestrin.

We have also been engaged in synthetic studies in this group for some time and therefore, in the hope of avoiding duplication of research, we take an early opportunity of indicating the lines on which we are proceeding.

The method is similar in principle to that of Braun and Irmisch ${ }^{2}$ but the stages are effected in a different manner and the yields of pure products have been greatly improved.

$\beta: \beta^{\prime}$-Diphenyladipic acid (i) (meso- and rac-), is accessible in several ways, for example, by reduction

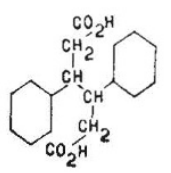

(i)

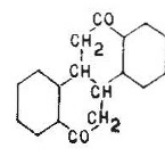

(ii)

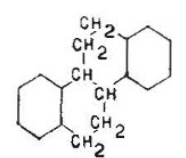

(iii)

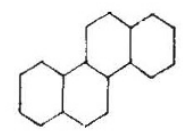

(iv) of ethyl cinnamate (or benzylidenemalonic ester, etc.) by means of aluminium; it suffers twofold dehydration on treatment with hot 85 per cent sulphuric acid giving the diketohexahydrochrysenes (ii) (cis- and trans-). Reduction of the cis-diketone by Clemmensen's method affords a hexahydrochrysene (iii) the constitution of which is confirmed by its dehydrogenation by selenium to chrysene (iv).
Starting with the appropriate substituents in the benzene rings (possibly $\beta: \beta^{\prime}$-dianisyladipic acid will serve the purpose) we can see our way, as clearly as any paper-synthesis allows, to the preparation of a norketohydroxyœstrin, that is, the natural product without its angle-methyl group.

\section{G. R. RAMAgE}

R. Robinson.

The Dyson Perrins Laboratory,

S. Parks Road,

Oxford.

Jan. 31 .

1 Nature, 131, 56, Jan. 14, 1933 .

2 Berickte, 64, 2461; 1931.

\section{Sex Hormones and Cancer-Producing Compounds}

A SEQUEL to the formulation of the sterols and bile acids as hydrogenated phenanthrene derivatives ${ }^{1}$ was the suggestion ${ }^{2}$ that carcinogenic derivatives of 1 : 2-benzanthracene might arise by abnormal sterol metabolism. No experimental evidence for ringclosure to the hydrogenated benzanthracene ringsystem has yet been put forward. Whether this actually occurs or not, it seems probable that the formation of a carcinogenic compound from a sterol would involve dehydrogenation to the aromatic state, for reduction of $1: 2: 5: 6$-dibenzanthracene to an octahydro compound results in loss of carcinogenic activity. Recent investigations of the ovarian hormones support the view that such sterol dehydrogenations do occur in the animal body. Thus oestrin is a tetracyclic compound which contains one aromatic ring, and Girard ${ }^{3}$ has recently isolated from the urine of pregnant mares an cestrogenic compound which undoubtedly contains two aromatic rings.

For reasons such as these, and because the cell proliferation which characterises the œstrous state is in some respects reminiscent of the early stages of a malignant growth, we have sought a correlation between substances having cestrogenic action and those having carcinogenic properties. Four main lines of attack have been initiated. These are :(a) The production of synthetic compounds of known constitution which possess œstrogenic properties. One such substance, 1-keto-1: $2: 3: 4$-tetrahydrophenanthrene, has already been described ${ }^{4}$ (b) The examination for œestrogenic activity of compounds containing the same ring-system as carcinogenic hydrocarbons, but with polar groups present and with some rings partially reduced. One such compound, $9: 10$-dihydroxy-9:10-di-n-butyl-9: 10 -dihydro-1 : $2: 5: 6$-dibenzanthracene ${ }^{5}$ has now given positive results. (c) In addition, we have found ostrus-exciting activity to be possessed by the two most potent carcinogenic hydrocarbons yet known, namely, 5 : 6 -cyclopenteno-1 : 2-benzanthracene ${ }^{6}$ and 1 : 2-benzpyrene? . We confess that this last result was entirely unexpected; it is very striking that both types of biological activity should be shown by one and the same compound. (d) The examination for carcinogenic activity of cestrin and related compounds, especially the basic aromatic hydrocarbon 1 : 2-cyclopentenophenanthrene, is being undertaken.

The technique of testing for cestrous activity was that described in Nature of January 14. The difficulties of solubility of the three compounds reported was very much greater than was experienced hitherto, and in all three cases $100 \mathrm{mgm}$. of substance was injected in a suspension in sesame oil. It is impossible to obtain any idea of the potency of the substances 